\title{
Igualdad, diferencia y teoría feminista* Equality, Difference and Feminist Theory
}

\author{
Natalina Stamile \\ Universidad de Bérgamo (Italia) \\ ORCID ID 0000-0002-7201-8539 \\ natalinastamile@yahoo.it
}

\section{Cita recomendada:}

Stamile, N. (2020). Igualdad, diferencia y teoría feminista. Eunomía. Revista en Cultura de la Legalidad, 18, pp. 9-28. DOI: https://doi.org/10.20318/eunomia.2020.5261

Recibido / received: 01/10/2018

Aceptado / accepted: 20/11/2019

\begin{abstract}
Resumen
Uno de los principales propósitos de este trabajo es analizar la compatibilidad entre la igualdad y la diferencia, dentro de la teoría feminista. En particular me interesa discutir el argumento desarrollado por algunas teóricas y feministas quienes afirman que la igualdad es compatible con las diferencias en el ámbito jurídico. Se acentuará el análisis a partir de la relación entre las Tecnologías de la Información y la Comunicación (TIC) y la condición de la mujer, buscando verificar si, a raíz de esta relación, ésta última sufre mudanzas considerables. En esta perspectiva, la introducción de la diferencia parece ser indispensable para alcanzar la igualdad y hacerla efectiva. Finalmente, se intentará defender que la contraposición teórica entre el feminismo de la igualdad y el feminismo de la diferencia, podría superarse proponiendo una forma de lectura que intenta conciliar las dos alternativas.
\end{abstract}

\section{Palabras clave}

Principio de igualdad, Estado de Derecho, diferencia, teoría feminista, TICs, conocimiento y desarrollo local.

\begin{abstract}
One of the main aims of this study is to analyze the compatibility between equality and difference in feminist theory. In particular, I am interested in discussing the argument of some theorists and feminists who affirm that equality and difference are compatible in the legal field. My analysis will focus on the relationship between Information and Communication Technologies (ICT) and the condition of women, verifying if, as a result of this relation, women suffer considerable changes. In this context, the introduction of difference seems to be indispensable to achieve equality and its effectiveness. I conclude that the juxtaposition of feminism of equality and feminism of difference could be overcome, and a form of lecture that tries to conciliate the two alternatives may be proposed.
\end{abstract}

\footnotetext{
* Un agradecimiento especial para Cleide Margareth Horban por revisar el texto y por los consejos sobre estilo además de su preciosa ayuda. También agradezco a Thomas Casadei, Ana Marcela Montanaro, Guadalupe Martin Dasso, así como a los revisores anónimos de este trabajo por haberlo leído y comentado y especialmente a Yarina Amoroso y a todos los organizadores y participantes del XV Congreso Internacional de Información, Info'2018, del 5 al 9 de marzo de 2018, celebrado en La Habana, Cuba, bajo el lema general: «Información y Conocimiento: desafíos para el Desarrollo Sostenible», por desarrollar reflexiones y debatir sobre el tema.
} 


\author{
Keywords \\ Equality principle, Rule of law, difference, feminist theory, ITC.
}

SUMARIO. 1. Introducción: Principio de Igualdad. 2. Teoría feminista. 2.1. El feminismo de la igualdad. 2.2. Entre igualdad y diferencia. 2.3. Feminismo de la diferencia. 3. Las Tecnologías de la Información y la Comunicación (TIC) y la condición de la mujer. 4. Conclusiones.

\title{
1. Introducción: Principio de Igualdad
}

El principio de igualdad es un tema clásico de la reflexión política y jurídica, que recién parece ser vuelto nuevamente de gran actualidad. Esto porque, en general, los últimos años se distinguen por una fuerte explosión de desigualdades sin precedentes en la historia. Esto, sin duda, pone en peligro no solo el principio de igualdad como fue formulado en todas las Constituciones, sino también la forma del Estado de Derecho y de la misma democracia. Desde siempre el principio de igualdad es considerado uno de los más importantes porque atrae en la lógica de su funcionamiento otras cláusulas generales de la Constitución. Ello explicaría por qué, este principio, fue definido como «uno de los momentos decisivos en el que hoy se manifiesta la pesadilla de las democracias» ${ }^{1}$.

El siempre mayor crecimiento de la discriminación y de la desigualdad parece ser influenciado por varios y diferentes factores. Por ejemplo, se piensa en la crisis del Estado social y de la esfera pública enfrente de la siempre más dominante globalización de la economía y de las finanzas o de los flujos migratorios y de las catástrofes ambientales que caracterizan cualquier lugar del mundo, sin considerar los innumerables ataques terroristas o todo lo que hace referencia al desplazamiento de un conflicto que toma el ciberespacio y las tecnologías de la información como campo de operaciones (guerra informática, guerra digital o ciberguerra).

Durante los últimos años, parece que, en un contexto particularmente teóricojurídico y no solamente social, el término crisis casi siempre está relacionado con el de emergencia, mejor con el concepto de Estado de emergencia (Cuono y Gargiulo, 2017, p. 17). Así, el binomio que los «espacios de crisis» requieren siempre «respuestas de emergencia» parece haberse convertido en una operación automática. En este contexto, propio el principio de igualdad, en toda su complejidad y pluralidad, asumiría un papel fundamental, mientras incluye las diferencias personales y al mismo tiempo excluye las diferencias sociales. En palabras de Pérez Luño,

Desde un punto de vista lógico, el concepto de igualdad significa la coincidencia o equivalencia parcial entre diferentes entes. Esta categoría es distinta de la identidad, que entraña la coincidencia absoluta de un ente consigo mismo, y de la semejanza, que evoca la mera afinidad o aproximación entre diferentes entes (2007, p. 17 [las cursivas son del autor]).

Así, se deduce que el principio de igualdad exigiría una pluralidad de personas, o de situaciones o de objetos, que pueden relacionarse y compararse entre ellos incluso a través un tertium comparationis. En otras palabras, y de manera sencilla,

\footnotetext{
${ }^{1}$ Véase Volpe (1977, p. X). La expresión es de Lambert.
} 
esto significa que el principio de igualdad puede asumir dos caras, es decir una formal y otra sustancial o una política y otra social. En el primer sentido, la igualdad consiste en atribuir un igual valor a todas las diferentes identidades que caracterizan un individuo como único y al mismo tiempo igual a los demás. En general, eso se podría traducir en el principio de no discriminación, dicho de otra manera, de tratar todos de manera igual, es decir sin discriminación y sin distinción, por ejemplo, en base al sexo, a la raza, a la lengua, a las opiniones políticas, a las condiciones personales y sociales. Por ejemplo, en su análisis Luigi Ferrajoli señala que

En este sentido, igualdad y diferencias no sólo no son antinómicas sino que se implican recíprocamente. [...] Y por eso vale para individualizar los confines tanto de la tolerancia, que reside en el respeto de todas las diferencias que forman las diversas identidades de las personas, como de lo que, al revés, reside en la inadmisibilidad de sus violaciones (1995, p. 906).

En la segunda acepción, la igualdad incluye la eliminación de todos los obstáculos, tanto de orden económico como social, que, de hecho, limitan la libertad y la misma igualdad y consecuentemente impiden el pleno desarrollo de la persona humana. Así, siempre en las palabras de Ferrajoli:

En este segundo sentido las diferencias, en lugar de ser rasgos de las diversas identidades de las personas, se convierten en privilegios o discriminaciones sociales que deforman la identidad y determinan la desigualdad de aquellas, perjudicando al mismo tiempo su igual valor. Por eso, conforme al propio principio de igualdad, son intolerables más allá de ciertos límites. Sólo que el confín entre tolerancia e intolerancia es en este caso bastante más problemático e incierto (1995, p. 906).

Sin embargo, la igualdad formal considera todas las personas como iguales mientras que la igualdad sustancial tiene en cuenta las diferencias, por ejemplo, sociales y económicas. Por ello, el principio de igualdad se caracteriza por ser un principio normativo y no descriptivo que implica necesariamente un juicio de valor y no un juicio de hechos. De acuerdo a este esquema, en el caso de la igualdad formal se generan diferencias y en el otro de la igualdad sustancial se producen desigualdades, de manera que

Unas deben ser reconocidas para ser respetadas y garantizadas; las otras deben serlo igualmente, pero para ser removidas o compensadas lo más posible. En todos los casos, la igualdad jurídica, tanto formal como sustancial, puede ser definida como igualdad en los derechos fundamentales. Los derechos fundamentales son, en efecto, las técnicas mediante las cuales la igualdad resulta en ambos casos asegurada o perseguida; y es la diversa naturaleza de los derechos sancionados en los dos casos lo que permite explicar su diverso modo de relación con las desigualdades de hecho (Ferrajoli, 1995, p. 907 [las cursivas son del autor]).

Por lo tanto, la igualdad formal afirmaría una igualdad en las diferencias convirtiendo la diversidad como un valor a garantizar. Por otro lado, la igualdad sustancial se basaría en la posibilidad de compensar las desigualdades y en este caso la diversidad se caracteriza con un desvalor a oponerse y eliminar. Además, es importante subrayar que la relación entre la igualdad y los derechos fundamentales se caracteriza para ser biunívoca, es decir que tanto la igualdad es constitutiva de los derechos fundamentales cuanto los derechos fundamentales son constitutivos de la igualdad (Ferrajoli, 1995, p. 908). Así, el problema de la igualdad en los derechos fundamentales radica en mostrar que existen diversas maneras alternativas de cumplir esa función normativa del principio de igualdad. Por ello, el propio principio de igualdad se ligaría, tal vez casi a confundirse, con el principio de razonabilidad. Por ejemplo, la igualdad sería violada todas las veces que una disciplina prescriba un tratamiento diferente o prevea diferencias que se caracterizan por ser totalmente 
irrazonables o sin ninguna base racional. Sin embargo, a pesar de la intuitiva importancia de esta función existe un profundo desacuerdo acerca del papel que la razonabilidad desempeña en nuestros sistemas jurídicos y normativos o, en general, en el derecho. Sin duda, una revisión exhaustiva de la igualdad y la razonabilidad daría lugar a una obra de considerable extensión y complejidad. Entre otras cosas, esto significa que aquí dejaré abierto el problema de qué tipos de relaciones y vinculaciones y, si existen diferencias significativas entre el principio de igualdad y la razonabilidad que se producen en el marco de un sistema jurídico (Stamile, 2015, pp. 11-24). Por ello, en este trabajo, restringiré el análisis a algunos problemas vinculados a la compatibilidad entre la igualdad y la diferencia, dentro de la teoría feminista. En la primera parte, en particular me interesa discutir el argumento desarrollado por algunas teóricas y feministas quienes afirman que la igualdad es compatible con las diferencias en el ámbito jurídico. Esta tesis se basa en la concepción del «dilema de la diferencia»; es decir, el «dilema de la diferencia», como momento crítico en la teoría del derecho, parte del surgimiento de la diferencia como un nuevo elemento en un proceso de problematización de la condición social de la mujer. En la segunda parte, se acentuará el análisis a partir de la relación entre las Tecnologías de la Información y la Comunicación (TIC) y la condición de la mujer, buscando verificar si, a raíz de esta relación, ésta última sufre mudanzas considerables. El mundo de hoy parece siempre más dominado por internet y la tecnología. Es bastante habitual y frecuente utilizar dispositivos que nos permiten acceder a internet. Sin embargo, por diferentes razones, no todas las mujeres tienen la misma posibilidad de acceder a las TIC. En esta perspectiva, la introducción de la diferencia parece ser indispensable para alcanzar el corazón de la igualdad (también y no solo a partir del conocimiento que incluye a las TIC), y consecuentemente hacerla efectiva. Finalmente, se intentará defender que la contraposición teórica entre el feminismo de la igualdad y el feminismo de la diferencia, que surgió principalmente en América, podría superarse proponiendo una forma de lectura que intenta conciliar las dos alternativas, puesto que el extremismo de las dos tesis solo funcionaría en el nivel teórico, ciertamente no en el nivel práctico-real.

\section{Teoría feminista}

El feminismo se caracteriza por lo que comúnmente se llaman las «olas feministas». Por ello, se distingue una primera ola, basada en el pensamiento de igualdad, una segunda ola ${ }^{2}$ sobre el pensamiento de la diferencia y, finalmente, una tercera ola definida como el feminismo posmoderno (Cavarero y Restaino, 2002). En otras palabras, es frecuente referirse a un feminismo liberal, a un feminismo cultural y a un feminismo radical (Grant Bowmany Schneider, 1998); o a un feminismo liberal o de la igualdad o de la paridad, _porque el objetivo principal es la igualdad entre hombres y mujeres ante la ley-, a un feminismo anarquista y libertario $y$, en último, a un feminismo radical (Facchi, 2007). Las diferentes etiquetas del feminismo, así como las diferentes demarcaciones temporales de las varias olas han creado y continúan alimentando un animado debate que parece destinado a no alcanzar una posición unívoca. Pero, a partir de lo que sostiene Kymlicka, que también plantea como premisa que la política feminista contemporánea es extremadamente variada, sería posible identificar algunas variaciones principales del feminismo, es decir: el feminismo liberal, el socialista y el libertarista (Kymlicka, 1995, pp. 259-315). Además, de particular importancia es la clasificación formulada por Moira Gatens (1991) que se basa en la relación entre el feminismo y algunas de las otras teorías socio-políticas

\footnotetext{
2 Nicholson (1997). Ya desde el título de la obra, está claro cómo la autora sea la primera en referirse a una segunda ola de feminismo que se centra en cuestiones relacionadas con la sexualidad y la esfera de la reproducción.
} 
como el igualitarismo, el liberalismo, el utilitarismo, el existencialismo, el marxismo y el psicoanálisis. Más aún, independientemente de las diferentes clasificaciones, según Alison Jaggar (1983), la base común del feminismo debe reducirse al objetivo de eliminar la subordinación de las mujeres; sin embargo, esta convergencia se disolvería si se prestara atención a las diversas articulaciones y concepciones de las mismas y a las relativas soluciones a su eliminación ${ }^{3}$. Por ello, al comentar este aspecto, Kymlicka señala que, aunque no falta una parte de la filosofía política que ha tolerado, defendido y aceptado la discriminación en razón del sexo, también es importante subrayar que «un amplio número de teorías políticas contemporáneas comparte una "plataforma igualitaria", un compromiso con la idea de que todos los miembros de la comunidad deberían ser tratados como iguales» (1995, p. 259).

\subsection{El feminismo de la igualdad}

La primera ola del feminismo se caracteriza por plantear el problema de la igualdad entre los dos sexos. El objetivo es, por tanto, eliminar todas las formas de discriminación entre hombres y mujeres basadas en razón del sexo. Tradicionalmente a las mujeres se les atribuían características que las colocaban en una clara subordinación e inferioridad en relación a los hombres, esto llevó a su exclusión de algunos contextos, ya que se les consideraba inadecuadas. Así, la afirmación de estas primeras feministas gira en torno a conceptos como igualdad y emancipación. Los hombres y las mujeres deben tener los mismos derechos, pero esto es poco probable que suceda en una sociedad patriarcal en la que el papel dominante pertenece al hombre mientras que a la mujer se reserva el de inferioridad, con la consecuencia de que no se pueden reconocer la igualdad de derechos y oportunidades si no hay igualdad entre las diversas posiciones mantenidas. El pensamiento feminista, por lo tanto, reclama la paridad, por ejemplo, en el nivel de educación y de derechos civiles fundamentales, comenzando a denunciar aquellas situaciones en las cuales surge una diferencia injustificada entre hombres y mujeres.

Como emblema de esta lucha puede considerarse el trabajo de Mary Wollstonecraft, Vindicación de los derechos de la mujer, publicado en 1792 con el título original en inglés $A$ Vindication of the Rights of Woman: with Strictures on Political and Moral Subjects. La estrategia argumentativa utilizada es la del pensamiento liberal y democrático que será la base de toda la primera ola de feminismo que intenta contrastar la visión de la inferioridad natural de las mujeres respecto a los hombres. Por ejemplo, en Italia, y especialmente en Francia, la igualdad se percibe como un principio ético-político que debe superarse, ya que puede borrar las diferencias y ocultar la exclusión femenina de la ciudadanía (Mancina, 2002, p. 6). Desde este punto de vista, se comprende mejor por qué el sufragismo, en el reclamo de la emancipación feminista, a través del sufragio universal, inicia la discusión sobre la renovación de los fundamentos morales de la sociedad. Así, el debate de esa época se concentraba acerca de la cuestión de que el derecho al voto debía reconocerse solo a quienes tenían una conducta moral responsable. Las sufragistas, entonces, argumentaron basándose en la diferencia femenina, es decir, que el derecho al voto también debería ser reconocido a las mujeres por su superioridad moral dada por la función materna y su compromiso humanitario en contra de los vicios y la corrupción moral (Evans, 1977). Por lo tanto, el primer feminismo se ha construido también alrededor y con referencia a la diferencia sexual (Facchi, 2004, p. 237). Según Susan Moller Okin (1989), sin embargo, la igualdad no debe confundirse con la identidad

\footnotetext{
3 Jaggar (1983). La autora distingue un feminismo liberal, socialista y radical.
} 
(sameness) y debe oponerse a las desigualdades y no a las diferencias ${ }^{4}$. En esta perspectiva, la raíz de la desigualdad entre hombres y mujeres en la sociedad se radica en la distribución desigual del trabajo dentro de la familia, considerada como un lugar privilegiado para la reflexión política. De hecho, los niños comenzarían a aprender en sus hogares que la interacción entre las personas no ocurre de acuerdo con modelos de igualdad y reciprocidad, sino a través de un esquema basado en la dependencia y la subordinación. Por ello, que tanto Susan Moller Okin (1989) como Martha Nussbaum (2002) enfatizan un aspecto relevante en este enfoque, es decir, que la familia patriarcal enseña a los niños la naturalidad (naturalness) de la subordinación que daña ciertos ideales liberales como, por ejemplo, el de la educación moral y de la democracia ${ }^{5}$. Las dos estudiosas coinciden en que la mayoría de los estudios llevados a cabo por destacados teóricos y académicos adoptan, como punto de partida, una perspectiva no neutral sino masculina, asumiendo que la institución de la familia se presenta como «justa». Por ello, al comentar este problema, Susan Moller Okin señala una consideración más: la familia perpetúa la desigualdad de género que se extiende por toda la sociedad. De acuerdo con este análisis, los niños adquirirían los valores y las ideas sexistas en el ambiente familiar en el que crecen, y terminando casi con seguridad, manteniéndolos en su vida adulta.

Esta es la base desde la cual Susan Moller Okin comienza a criticar la teoría de la justicia formulada por Rawls, que no estaría completa porque no incluiría a las mujeres y no tendría en cuenta adecuadamente las desigualdades de género originadas en la familia.

Es interesante notar que la familia en los estudios con perspectiva de género asume una posición central, porque es considerada el lugar por excelencia de la educación cultural y social de las generaciones futuras y consecuentemente es el lugar concreto de intervención. Entre otras cosas, esto significa, también que es el punto de partida de los varios procesos de reelaboración cultural de la sociedad y la fuente de cualquier hipótesis normativa dirigida a la reconstrucción de la justicia social en general. Así, la familia ha sido definida por las feministas como un sinónimo de la esfera doméstica y privada en la que a las mujeres se les ha atribuido tradicionalmente características sociales de inferioridad, determinadas solo por una diferencia sexual. Una de las causas se individúa en la estructura de las relaciones sociales y económicas familiares, concebidas para crear dependencia de algunos miembros, las mujeres, respecto a otros, los hombres. Sin embargo, la familia podría considerarse el lugar originario para plantear la cuestión de género, mientras no puede existir una sociedad justa si, en general, la familia como ámbito puramente privado no venga reconducida también a un problema político. En otras palabras, se debería considerar la familia como parte integral y lógicamente prioritaria de la teoría de la justicia en la sociedad, porque una familia injusta produce una sociedad injusta y hace que las personas, sean inadecuadas a formar una sociedad justa.

\subsection{Entre igualdad y diferencia}

Muchas teóricas feministas han intentado analizar el problema de la esfera política y luego abordar el tema de la justicia en relación con las mujeres, abriendo la discusión sobre las relaciones complejas que surgen entre el feminismo y la justicia, las perspectivas de género y las diferencias culturales. Es interesante notar que el modelo

\footnotetext{
${ }^{4}$ La posición de Susan Moller Okin, parece ser compartida también por Scott (1988), la cual argumenta que el antónimo apropiado de la igualdad es la desigualdad, no la diferencia, y el de la diferencia es identidad, no igualdad.

${ }^{5}$ Nussbaum (2002, pp. 409-500), en particular, afirma que «the patriarchal family may teach children a lesson in the «naturalness» of subordination that undermines other liberal ideals such as moral education and democracy».
} 
de igualdad liberal opera dentro del paradigma jurídico liberal, permaneciendo así involucrado en todas las críticas que se dirigen a este último. Por lo tanto, es fuertemente criticada la mera igualdad nominal, garantizada dentro del liberalismo más tradicional, y se sostiene la necesidad de garantizar una igualdad, que sea real para las mujeres, posible solo mediante la revisión de ciertos valores a la luz de consideraciones de género ${ }^{6}$. Por ello, al comentar este problema, Stein es más incisiva y señala que

this vision wholly ignores the sexual oppression that leads to unwanted pregnancy, oppression that takes many forms from rape to the social conditioning that makes women unable to say no, or, worse, unable even to want to say no (1993, p. 1166),

con la consecuencia de que todo lo que es concerniente al mundo femenino parecería ser totalmente ignorado. De la misma posición es Carol Gilligan (1982), que elabora la expresión different voice con el objetivo de destacar cómo las mujeres resuelven, de forma completamente diferente de los hombres, algunos de los principales dilemas morales. Así, el razonamiento moral masculino hace referencia a los derechos abstractos o a una ética de la justicia -ethic of justice-, mientras que lo de las mujeres se desarrolla acerca del concepto de responsabilidad de sí mismo y de los demás, es decir una ética del cuidado (ethic of care). Además, Gilligan precisa que, por un lado, la ética de la justicia se basa en el concepto de igualdad y de equidad del juicio, es decir reconociendo el igual respeto para todos y buscando una forma de equilibrio entre las exigencias de los otros y las propias. Por otro lado, la ética de la responsabilidad se funda acerca del concepto de justicia distributiva, implicando el reconocimiento y la comprensión de la diversidad de las necesidades que dan lugar a una compasión y al cuidado (Gilligan, 1982, p. 166).

Por lo tanto, la pregunta que debe hacerse es qué significa realmente la igualdad y si reconocer el valor de las diferencias puede afectar negativamente a la misma. Algunas teóricas feministas se concentraron en el análisis de la relación, no siempre unívoca, entre la igualdad y las diferencias. Luego de una inicial pretensión de no discriminación e igualdad, el pensamiento feminista parece vivir un cambio en términos de libertad de las mujeres, es decir que

punto crucial de esta inflexión es la idea de que las mujeres no obtendrán la libertad adhiriéndose al modelo tradicional de sujeto libre, pensado neutro pero en la realidad masculina, sino que para ganar libertad sea necesario repensar las mismas estructuras de pensamiento, para que puedan permitir a las mujeres la libertad de dar forma a sí misma de acuerdo a todas las diferencias que cada una encuentra significativas ${ }^{7}$.

Por lo tanto, en la segunda ola del feminismo, el debate parece enfocarse en la diferencia sexual, que ya no se considera un mero dato natural, sino que se convierte en un concepto que debe justificarse, para combatir una forma persistente de discriminación. De esta manera, se empieza a construir las bases para el desarrollo del concepto de género (gender) y a estructurar la dicotomía entre sexo y género. Sin duda, el feminismo liberal parece cerrarse en sí mismo y entra en crisis cuando no va

\footnotetext{
${ }^{6}$ Véase, por ejemplo, Fineman (2005, p. 19) que señala: «this model focuses on ensuring that women are afforded genuine equality as opposed to the nominal equality often given them in the traditional liberal framework and seeks to achieve this either by way of a more thorough application of liberal values to women's experiences or the revision of liberal categories to take gender into account». Véase, además, las obras y reflexiones desarrolladas por H. T. Mill, J. S. Mill y B. Friedan, entre otras.

${ }^{7}$ C. BotTI, Etica e differenza di genere en:

http://www.treccani.it/scuola/tesine/maschile e femminile/2.html. La autora utiliza entre comillas una afirmación de G. Bock, S. James (1992), Beyond Equality and Difference, Routledge, London-New York, p. 7.
} 
más allá de la igualdad formal (Morondo Taramundi, 2004, p. 28). Por ello, al comentar este problema, Dolores Morondo Taramundi señala que, durante el siglo XIX y la primera mitad del siglo XX, el feminismo liberal luchaba por la igualdad de derechos para las mujeres, la igualdad de acceso a las profesiones y a la participación política. Estas pretensiones se basaban en la igual capacidad de las mujeres de ocuparse de esas actividades, así como en su naturaleza igualmente racional y razonable (2004, pp. 28-29). A pesar de esto, no faltaron argumentos para sostener que la igualdad es compatible con las diferencias en el ámbito jurídico (Gianformaggio, 1993). Por ejemplo, Joan W. Scott notaba que, especialmente en los años setenta, el feminismo consideraba la diferencia como «el instrumento analítico más creativo» (1995, p. 271). Así que, recién dentro de la teoría feminista se está comenzando a desarrollarse la concepción del dilema de la diferencia, es decir, como momento crítico en la teoría del derecho, parte del surgimiento de la diferencia como un nuevo elemento en un proceso de problematización de la condición social de la mujer (Morondo Taramundi, 2004, p. 7). En esta perspectiva, la introducción de la diferencia parece ser indispensable para alcanzar el corazón de la igualdad —-también y no solo a partir del conocimiento que incluye a las TIC como se verá luego-, y consecuentemente hacerla efectiva. La contraposición teórica, entonces, entre el feminismo de la igualdad y el feminismo de la diferencia, que surge principalmente en los Estados Unidos, podría superarse proponiendo una clave de lectura que pueda conciliar las dos alternativas ${ }^{8}$.

Punto de partida es considerar la igualdad y la diferencia no como dos conceptos en real oposición normativa porque:

when equality and difference are paired dichotomously, they structure an impossible choice. If one opts for equality, one is forced to accept the notion that difference is antithetical to it. If one opts for difference, one admits that equality is unattainable (Scott, 1995, p. 271).

De manera que el feminismo no puede abandonar, por una parte, la diferencia, porque como se decía antes es el instrumento analítico más creativo, y por otra parte la igualdad, porque todas las feministas necesitan de la igualdad para comunicar en modo inteligible con los principios y valores de nuestras sociedades (Morondo Taramundi, 2004, p. 83). Por lo tanto, la oposición igualdad-diferencia habría generado un dilema solo en el nivel lingüístico. Por ejemplo, según la lectura de Letizia Gianformaggio, la igualdad y la diferencia deben considerarse complementarias, como dos caras de la misma moneda (Gianformaggio, 1993, 1995 y 1996). Su tesis se basa en que se puede predicar la igualdad o la diferencia en tres niveles: (i) descriptivo, (ii) prescriptivo y (iii) evaluativo (Gianformaggio, 1993, p. 218). Sin embargo, la identificación de (i) un nivel descriptivo y (ii) de un nivel prescriptivo ciertamente no es una novedad, ya que, desde Aristóteles, se afirmó que la igualdad y la diferencia son conceptos relacionales. En las palabras de Letizia Gianformaggio el problema sería el siguiente:

[...] se refieren a la relación de dos elementos distintos según un estándar de comparación. La igualdad, descriptiva o prescriptiva, es una relación transitiva y recíproca. La diferencia, descriptiva o prescriptiva, es recíproca pero no transitiva. La igualdad es recíproca porque si $A$ es igual a $B$, entonces $B$ es igual a $A$. Es transitivo porque si $A$ y $B$ son iguales y $A$ y $C$ son iguales, entonces $B$ y $C$ también son iguales. Por el contrario, la diferencia es recíproca, porque si $A$ es diferente de $B$, entonces $B$ es diferente de A; no es pero transitiva. Si A y B son diferentes, y A y $C$ son diferentes, no es necesariamente cierto que B y $C$ son diferentes (1993, p. 218ss.).

\footnotetext{
${ }^{8}$ Véase, para una mayor profundización las varias obras de Martha Minow y Joan W. Scott.
} 
De esto sigue, que la igualdad puede atribuirse solo a dos elementos iguales en el sentido de que pueden ser diferentes y similares en algunos aspectos, mientras que la identidad solo puede realizarse en referencia a sí misma, es decir, A es idéntica a $A$ con referencia al carácter (iii) evaluativo, la autora subraya que: decir que «A es diferente de $B$ » no es lo mismo que decir «B es diferente de $A$ » o decir «A y $B$ son diferentes» (Gianformaggio, 1993, p. 221). Esto, porque A y B no se consideran iguales o diferentes según tengan o no una característica común, como ocurre a nivel (i) descriptivo y (ii) predicativo, sino que A y B se comparan entre sí asumiendo $B$ como el estándar de la misma comparación. Además, Letizia Gianformaggio elabora el concepto evaluativo de la igualdad y de la diferencia. Por una parte, la igualdad no está acompañada de la idea de relación de acuerdo con una cierta medida, sino por la idea de valer cuanto y de ser tan bueno como. Por otra parte, la diferencia evaluativa está marcada por las connotaciones negativas de no alcanzar el estándar de, es decir, por la inferioridad (Gianformaggio, 1993, p. 222). En esta última (iii) dimensión evaluativa, la comparación se realiza mediante un tertium comparationis que es interno a la misma relación entre igualdad y diferencia y no es un elemento externo, como ocurre en la dimensión (i) prescriptiva y (ii) descriptiva. Sin embargo, a pesar de la intuitiva importancia de esta estrategia, existen críticas formuladas por las feministas y un profundo desacuerdo acerca del papel que desempeñan como presupuesto lo que comúnmente se define como «norma de comparación masculina o estándar masculino de excelencia» (Morondo Taramundi, 2004, p. 108). Desde este punto de vista, el derecho sería neutral solo aparentemente, en cuanto compuesto de normas masculinas se convertiría solo en un instrumento adicional de dominación de la sociedad machista. Por ello se señala que:

por ejemplo, cuando las mujeres piden iguales derechos o paridad de tratamiento, la referencia implícita son los hombres (o un determinado grupo de hombres). Pero también cuando sostienen ser diferentes y piden derechos diferenciados, las mujeres están usando a los hombres como un estándar implícito: son diferentes de los hombres y quieren derechos diferenciados de los derechos de los hombres (Morondo Taramundi, 2004, p. 112) ${ }^{9}$.

Según otra perspectiva de análisis, parece que las feministas de la diferencia cuestionan no tanto el valor de la igualdad en sí misma (porque la igualdad y/o el universalismo no es algo separado y aislado respecto a la diferencia sexual) sino la concepción de igualdad desarrollada dentro de la tradición liberal. Por ejemplo, Luigi Ferrajoli, en su análisis teórico del significado del principio de igualdad y su relación con la diferencia de género, precisa que es necesario tener en cuenta que

la igualdad [...] es un principio complejo, estipulado para proteger las diferencias y en contra de las desigualdades. Diferencias y desigualdades son términos que designan conceptos diversos, pero ambos dotados de un estatus diferente y asimétrico con respecto al del término igualdad (2015, p.1).

Por lo tanto, se podría afirmar que cada diferencia, así como aquellas sexuales, "consisten en la diversidad de identidades personales que hacen que cada uno sea diferente del otro» (Ferrajoli, 2015, p. 1). Esta definición se referiría a la igualdad formal, es decir que todas las personas deben ser tratadas igualmente ante la ley; mientras que las desigualdades, entendidas como todas aquellas diferencias que se derivan de las condiciones económicas y materiales de cada individuo, formarían a la igualdad sustancial. Entonces, el principio de igualdad sería, sin duda, un principio normativo compuesto de dos niveles: uno dirigido a proteger y mejorar

\footnotetext{
${ }^{9}$ La autora subraya que a este respecto la literatura no es solo enorme, sino que tampoco se presenta de manera unívoca ni desarrollada adecuadamente desde una perspectiva puramente normativa y de la teoría del derecho.
} 
las diferencias, incluso las de género, y el otro, dirigido a reducir y/o eliminar las desigualdades. Por ejemplo, en su análisis del principio de igualdad en la relación con la diferencia de género, Luigi Ferrajoli señala que:

el principio de igualdad en este primer significado como el igual valor asociado a todas las diferencias de identidad que hacen a cada persona un individuo diferente de todos los demás y de cada individuo una persona como cualquier otra $\left(2015\right.$, p. 1) ${ }^{10}$,

y además,

en el igual valor asociado a las diferencias, la igualdad consiste también en el desvalor asociado a todas las desigualdades materiales y sociales de las cuales el igual valor de las diferencias y la igual dignidad de las personas son de hecho limitadas o, lo que es peor, negadas $(2015, \text { p. } 2)^{11}$.

De esta manera, igualdad y diferencia no serían en contraposición, sino dos principios complementarios. En este sentido, el argumento desarrollado por Luigi Ferrajoli se condensa de la siguiente manera:

la contradicción es solo entre igualdad y desigualdad. La igualdad, en resumen, es un principio complejo que impone la protección de las diferencias y la reducción de las desigualdades y que realiza esta su dúplice valencia normativa gracias a su nexo lógico con el universalismo de los derechos fundamentales: de los derechos de libertad para proteger a los iguales valores de las diferencias; de los derechos sociales contra las desigualdades materiales y sociales $(2015$, p. 3$)$.

A efectos de lograr la mayor claridad posible, hay que subrayar que Ferrajoli identifica cuatro formas de entender el tratamiento jurídico de las diferencias y entonces también el de la igualdad: (i) indiferencia jurídica para las diferencias; (ii) la diferenciación jurídica de las diferencias; (iii) la homologación jurídica de las diferencias, y (iv) la valoración jurídica de las diferencias (Ferrajoli, 1993, pp. 50-53). Esta estrategia permitiría alcanzar la conclusión que la igualdad es igualdad en los derechos fundamentales (Ferrajoli, 2015, p. 6).

Esta interpretación, —especialmente en Francia e Italia-ciertamente no está entre las más comunes; porque en estos países el feminismo de la diferencia está más firme y adopta una definición tradicional de igualdad en un sentido descriptivo (la igualdad es un hecho) en lugar de considerar la igualdad en un sentido normativo (es decir, como un valor $)^{12}$.

\subsection{Feminismo de la diferencia}

Las reflexiones sobre la diferencia, sin duda, abren nuevos escenarios para el feminismo (Cavarero y Restaino, 1999). Por ejemplo, en los Estados Unidos, tales reflexiones encuentran terreno fértil, especialmente en aquellos movimientos feministas que tienden a valorizar las diferencias agregándolas a la igualdad. Por ejemplo, Iris Marion Young, considerada una de las pioneras del pensamiento de la diferencia, señala que:

\footnotetext{
10 (Cursiva del autor).

11 (Cursiva del autor).

12 Para un análisis del feminismo de la diferencia, véase, por ejemplo: Lonzi (1974); Irigaray (1974); Libreria delle donne di Milano (ed.) (1987); Cavarero (1987); Muraro (1991); Bono y Kemp (1991); Restaino (1999); Cavarero y Restaino (1999) en particular las págs. 73-81 y 101-110; Boccia (2002).
} 
Apruebo esta política de la diferencia, y sostengo que lo que está en cuestión es el propio significado de diferencia social. La política tradicional que excluye o desvaloriza a algunas personas por sus atributos de grupo asume un significado esencialista de la diferencia; define a los grupos como portadores de naturalezas diferentes. Una política igualitaria de la diferencia, en cambio, define la diferencia de manera más fluida y relacional, como el producto de procesos sociales. Una política emancipatoria que afirme la diferencia de grupo conlleva la recepción del significado de igualdad. El ideal de asimilación asume que la igual categoría social de todas las personas requiere tratar a todas ellas de acuerdo con los mismos principios, reglas y criterios. Una política de la diferencia sostiene, en cambio, que la igualdad como participación e inclusión de todos los grupos requiere a veces un tratamiento diferente para los grupos oprimidos o desaventajados (2000, p. 266).

En particular, en esta etapa, las feministas se centran en demostrar la carencia de fundamento de la tesis de la universalidad y de la neutralidad de la concepción liberal-democrática de la justicia basada, aunque solo parcialmente, en una concepción masculina. Por ejemplo, una crítica eficaz está basada en contra de la falsa neutralidad que las sociedades liberales presentarían. En general, en la construcción de teorías, en la afirmación de derechos y en la elaboración de normas, la referencia es, por ejemplo, a un sujeto neutral, sin raza, sexo, clase social, etcétera, por lo menos desde que la igualdad se ha impuesto como principio universal. Pero, este sujeto, en la realidad, tiene características precisas que normalmente corresponden a las del grupo dominante; así tomarlo como modelo implica la exclusión o, en cualquier caso, la discriminación de los otros individuos (Facchi, 1999, p. 131). Esta perspectiva de análisis parece estar influenciada por los distintos estudios sobre el sexo y el género. Por un lado, con el primer término, (es decir, sexo) se determina la diferencia biológica entre un hombre y una mujer. Por otro lado, el género indica los papeles sociales impuestos por la dominación patriarcal o machista, respectivamente, a las mujeres (subordinación en todos los campos) y al hombre (dominio en todos los campos) (Abbagnano, 2012, p. 471). Hay que subrayar que este aspecto se releva determinante porque permite destacar cómo el feminismo liberal, desarrollado especialmente en los años sesenta y setenta, lucha por los derechos individuales de las mujeres, pero no a través de una perspectiva de género. Además, es importante añadir que el feminismo liberal no tiene como objetivo cambiar la estructura social, sino que simplemente se limita de proponer a implementar las acciones positivas y la igualdad de las oportunidades para eliminar la discriminación entre mujeres y hombres. Por lo tanto, el objetivo es dar visibilidad a las mujeres, pero en el mismo sistema o en la misma estructura de poder, a pesar de sus limitaciones y deficiencias. Tal vez esto sería uno de los límites del feminismo liberal: la reivindicación del papel de la mujer pasa por la no ruptura del modelo existente de la sociedad o de la familia que crea un sujeto-mujer falsamente universal y neutral. Por ello, al comentar este problema, Catherine MacKinnon (1993) señala que la culpa del feminismo liberal es que ha protegido los derechos de las mujeres de manera demasiado abstracta, es decir, que no existe en su interpretación y aplicación alguna perspectiva de género. En sus palabras:

La doctrina dominante de la ley de discriminación sexual, que resulta de ellos es, en mi opinión, en gran medida responsable de que la ley de igualdad sexual haya sido tan escandalosamente ineficaz para darnos a las mujeres lo que necesitamos y socialmente se nos impide tener sobre la base de una condición de nacimiento: la posibilidad de tener vidas productivas con la seguridad física razonable, autoexpresión, individuación y un mínimo de respeto y dignidad (MacKinnon, 2014, p. $57)$.

Así, la discriminación sexual es descrita como una forma de desigualdad, porque en la perspectiva liberal, faltando la visión que la sexualidad es una forma de poder que define las relaciones de género, la propia sexualidad se traduce en 
relaciones de poder. De la misma manera, como analiza Kymlicka, las cosas quedan equivalentes y no cambian mucho, ni siquiera con referencia al feminismo de la diferencia, que tiene entre sus objetivos garantizar el acceso de las mujeres en contextos considerados típicamente masculinos, por ejemplo en las actividades manuales y militares, en el empleo público y etcétera, es decir en todos los lugares en los que las mujeres por su propia naturaleza estaban consideradas poco aptas para las tareas políticas y económicas fuera del hogar. En sus palabras:

Sin embargo, el éxito de este planteamiento ha sido limitado, porque desatiende las desigualdades que tienen su asiento en la misma definición de las posiciones mencionadas. La consideración de la diferencia ve la igualdad sexual en términos de la capacidad de las mujeres para competir, en un marco de reglas neutrales en lo tocante al sexo, por roles que han sido definidos por los hombres [...] (Kymlicka, 1995, p. 262).

En este enfoque, incluso si se ignora el sexo de los candidatos, a la hora de decidir quién podrá desempeñar un papel de prestigio y ocupar tales posiciones en las instituciones, será imposible alcanzar concretamente la igualdad si, en cualquier caso, se crean instituciones sociales que sean funcionales y conformes a los intereses de los hombres. Por ello, al comentar este problema, Kymlicka señala concluyendo que «el problema es que estos papeles pueden estar definidos de modo tal que los hombres resulten más aptos para ellos, incluso en un marco de reglas de competencia neutrales en materia de sexo» (1995, p. 263).

Entonces, el paradigma de la diferencia, parece declinarse en una doble forma. Por un lado, ha funcionado como un importante instrumento analítico para casi todos los grupos feministas pertenecientes a la segunda ola, incluidas las feministas de la igualdad. Por otro lado, ha funcionado, aunque de una manera más controvertida, como instrumento de reivindicación. Sin embargo, el contenido de las reivindicaciones de la diferencia o de las diferencias varía para cada uno de esos grupos que podríamos definir en general como feminismo de la diferencia (Morondo Taramundi, 2004, p. 4).

3. Las Tecnologías de la Información y la Comunicación (TIC) y la condición de la mujer

El análisis que desarrollaré a continuación asume ciertos presupuestos, a partir de las discusiones hasta aquí presentadas (aunque de manera sucinta), acerca del argumento desarrollado por quienes afirman que la igualdad es compatible con las diferencias en el ámbito jurídico. En particular, me interesa acentuar el análisis a partir de la relación entre las Tecnologías de la Información y la Comunicación (TIC) y la condición de la mujer, buscando verificar si, a raíz de esta relación, ésta última sufre mudanzas considerables ${ }^{13}$.

El mundo de hoy parece siempre más dominado por internet y la tecnología. Es bastante habitual y frecuente utilizar dispositivos como netbooks, tablets, smartphones, que nos permiten acceder a internet y múltiples aplicaciones para

\footnotetext{
${ }^{13}$ Esto significa que, en el presente estudio, no trataré lo que ha aportado el desarrollo a nivel nacional e internacional del derecho antidiscriminatorio, considerado este último como una de las claves para centrar la tensión igualdad/diferencias y para comprobar la evolución significativa que a nivel jurídico se ha producido en el contenido del principio de igualdad. Tampoco me centraré en el derecho antidiscriminatorio desarrollado por los Estados y las políticas públicas de igualdad. A este respecto véase: Casadei (2015).
} 
informarnos, comunicarnos, trabajar de forma totalmente nueva y al mismo tiempo más eficiente. Sin embargo, por diferentes razones, no todas las mujeres tienen la misma posibilidad de acceder a las TIC. Por ejemplo, Betty Friedan señala que algunos obstáculos que han limitado la participación de la mujer son las cualidades femeninas atribuidas por la sociedad. En general, las mujeres son reconocidas por una mayor capacidad de escucha, de comunicación, de empatía, de consenso y consecuentemente menor seguridad, que los hombres (Friedan, 2009). En esta perspectiva, la introducción de la diferencia parece ser indispensable para alcanzar el corazón de la igualdad, (y no solo se lograría a partir del conocimiento que incluye a las TIC), y consecuentemente hacerla efectiva.

En primer lugar, hay que evidenciar que los estudios y las aportaciones desde una perspectiva de género, que plantean unas cuestiones en relación a las Tecnologías de la Información y la Comunicación (TIC), son, sin dudas, recientes. En general e inicialmente, en muchas ocasiones, las teorías feministas se caracterizaron por mostrar un profundo escepticismo ante la histórica masculinidad inherente a la tecnología ${ }^{14}$. Solamente a partir de los años noventa comienza a surgir un interés de las mujeres por la tecnociencia en consideración de su potencial para transformar las relaciones de género tanto en una era digital y globalizada como en una sociedad de la información ${ }^{15}$. Por ejemplo, Donna Haraway, autora del Cyborg Manifesto (1991), elabora la metáfora del cyborg, definido como

un organismo cibernético, un híbrido de máquina y organismo, una criatura de realidad social y también de ficción [...]. La ciencia ficción contemporánea está llena de ciborgs - criaturas que son simultáneamente animal y máquina, que viven en mundos ambiguamente naturales y artificiales (1991, p. 2),

para argumentar que las máquinas pueden dar sus contribuciones a cambios relevantes en contra de las tradiciones de un capitalismo racista y de una sociedad dominada por los ideales masculinos y patriarcales. En este sentido Donna Haraway comenta que la personificación femenina parecía ser algo dado, orgánico, necesario así como la expresión de lo femenino parecía significar el disponer de habilidades maternales o en sentido estricto o metafórico. Solo estando fuera de lugar las mujeres pueden lograr un placer intenso con las máquinas y, entonces, con la excusa de que al fin y al cabo se trata de una actividad orgánica después de todo (Haraway, 1991).

En general, las ciberfeministas describen la era digital como un espacio donde las diferencias sexuales se diluyen, donde las mentes se liberan de sus cuerpos, donde su identidad no está sujeta ni determinada por su físico (Martínez Álvaro, 2016, p. 440). Así, la transformación de las relaciones entre las mujeres y las tecnologías se caracteriza por ser alcanzable y real y sobretodo como reacción al sistema patriarcal en las relaciones tecnológicas.

La sociedad ha cambiado y esta nueva sociedad ha provocado nuevas formas de producción, difusión y consumo del conocimiento y la cultura (Area, Gros y Marzal 2008). Así, es importante subrayar que

el conocimiento, es el capital de la sociedad y las TICs, las herramientas que nos ayudan a acceder y producir la información, por lo tanto, las TICs son unas herramientas que deben considerarse como algo más que soportes físicos transmisores de información, canales por los que vehicula información o contenedores

\footnotetext{
14 Por ejemplo, véase: Martínez Álvaro (2016), esp. p. 440; Harding (1998) la cual afirma que hay la existencia de múltiples investigaciones que han demostrado que la exclusión de las mujeres de la tecnología ha sido una consecuencia de la especialización de los oficios por hombres durante la revolución industrial. A este respecto véase también: Cockburn (1983); Stamile (2017).

${ }^{15}$ Véase por ejemplo Manuel Castells.
} 
de información, ya que al ser usadas por un individuo, existen variables inherentes a los sujetos que interaccionan con la información, variables del contexto de los usuarios y el método de enseñanza en el que se integran las TICs que tienen influencia en la información (Area 2009) ${ }^{16}$.

Algunos estudiosos distinguen tres dimensiones de las TIC: pedagógicas, de inclusión social y cultural (Area, 2009, p. 299); porque las TIC trasformaron y siguen trasformando de manera significativa nuestro lenguaje, nuestras formas de pensar, aprender, comunicarnos, trabajar, decidir y,

en fin, se caracterizan por su capacidad de modificar el curso y funcionamiento de las relaciones y las actividades humanas. Es justamente esa potencia disruptiva y sistémica lo que torna complejo, y al mismo tiempo decisivo, el proceso de la integración de las TICs en la educación (Area, 2009, p. 305).

Así que parece útil recordar la postura de Sheyla Benhabib que adhiere a una sociedad democrática deliberativa que permita un diálogo complejo entre varias culturas dentro de una esfera pública, es decir,

nacemos en redes de interlocución o redes narrativas, desde relatos familiares y de género hasta relatos lingüísticos y los grandes relatos de la identidad colectiva. Somos conscientes de quiénes somos aprendiendo a ser socios conversacionales en estos relatos (2006, p. 45).

Entonces las condiciones normativas serán reciprocidad igualitaria, autoadscripción voluntaria y libertad de salida y asociación con el fin de expresarse en una democracia deliberativa. Consecuentemente, adoptando esta perspectiva, las TIC dejan de ser simplemente un solo medio para convertirse en un sistema comunicativo que parece favorecer nuevas formas de subjetividades y relaciones. Por esa razón es importante tener siempre en cuenta que la discriminación de género está presente en un gran número de ámbitos (por ejemplo, laboral, educativo, acceso a la salud y participación política), con repercusiones profundamente perjudiciales para las sociedades en su conjunto. Además, cuando se discute específicamente sobre TIC, parece también relevante considerar que si

por un lado, es importante incrementar el uso de las TICs por las mujeres en términos cuantitativos, disminuyendo la brecha digital tanto de género como de ingreso, por el otro es necesario admitir que el mero acceso a las TICs no significa necesariamente que las tecnologías tengan un impacto positivo o empoderen las mujeres. Las TICs pueden ser una herramienta de apoyo y un instrumento de incentivación para crear o consolidar formas de diálogo, asociacionismo y cooperación para llevar al debate público on-line y off-line ${ }^{17}$.

Por mucho tiempo, el trabajo de la mujer en estos sectores ha sido clasificado como sub-profesionales, tanto por una cuestión de género como también para reducir los costos laborales. Y este problema parece persistir también ahora, si bien con intensidad diferente según la cultura y el país ${ }^{18}$. Por lo tanto, en los países más industrializados,

\footnotetext{
${ }^{16}$ Además, es importante subrayar que unas de las líneas de actuación prioritarias de la UE, tal como indica la Digital Agenda forEurope, es educar a los usuarios en las TIC y priorizar la alfabetización en las nuevas tecnologías.

17 V. Vilardo, Mujeres, TIC y transparencia - Tecnología - publicado en Mujeres en Red, http://www.mujeresenred.net/spip.php?article1762.

18 Véase Castells (2004), especialmente el 4 capítulo «El fin del patriarcado: movimientos sociales, familia y sexualidad en la era de la información», pp. 159-270.
} 
aunque el acceso a la tecnología y el uso de sus aplicaciones es cada vez más fácil e intuitivo, sigue existiendo una brecha digital de género que es "un problema» ya que la habilidad para utilizar Internet es cada día más necesaria, tanto para obtener mejores resultados académicos como para conseguir mejores puestos de trabajo y realizar mejor nuestra actividad laboral (Sosa, 2013).

Así que se distingue una primera brecha digital, es decir el acceso al ordenador y a la conexión de internet, que con el tiempo y en países desarrollados, ha ido desapareciendo. Una segunda brecha que afecta a los usos de las tecnologías de la información y comunicación, y está determinada por las capacidades y habilidades de los individuos para utilizar ordenadores (Castaño, 2008). Hay que subrayar que varios estudios demuestran que esta segunda brecha aflige sobre todo a las mujeres, mientras persistiría una preocupante ausencia de mujeres en algunos importantes ámbitos relacionados con las nuevas tecnologías ${ }^{19}$. Los factores pueden ser diferentes aunque relacionados con el concepto de patriarcado y de sub alteridad que aún también influyen sobre la elección de la mujer en este específico ámbito de la tecnología. Así, según algunos estudios es posible esquematizar estos factores de la siguiente manera:

la idea equivocada de la informática y de los estilos de vida de las personas de este ámbito, considerándose como ámbito que no es para mujeres, que consiste solo en programación, es muy difícil y hay poca interacción humana. La imagen estereotipada de los informáticos y del tipo de trabajo que desarrollan. Las pocas referencias femeninas en el ámbito. La poca confianza de las mujeres en sus destrezas con los ordenadores. Las familias, hacen que las chicas se sientan poco atraídas por estos estudios y no la consideren como carrera profesional a tener en cuenta (Fernández Sánchez, 2006, p. 466).

Es evidente como una cierta idea de sub alteridad y de modelo de sociedad patriarcal continúa reproduciéndose, aunque con diversas variantes. Así que surge la exigencia de establecer si el patriarcado, ante los distintos recursos de las tecnologías de la información y de la comunicación, dentro de una sociedad globalizada, todavía persiste o se podría hablar, aunque con debida cautela y prudencia de fin del patriarcado (por lo menos en algunas zonas más desarrolladas ${ }^{20}$ ), además teniendo

\footnotetext{
${ }^{19}$ Por ejemplo, en el Consejo de Europa, Conclusiones del Consejo de 5 de mayo de 2003 sobre los niveles de referencia del rendimiento medio europeo en educación y formación (Puntos de referencia) (2003/C 134/02) se afirma que «El equilibrio entre los sexos es un reto particularmente importante en esta área. El número de mujeres que optan por cursar estudios de licenciatura en matemáticas, ciencias y tecnología es relativamente menor que el de los hombres, y aún menos son las que se deciden por las carreras de investigación. Por lo tanto, el número total de licenciados en matemáticas, ciencias y tecnología [...] en la Unión Europea debería aumentar al menos en un 15 \% para 2010, al tiempo que debería disminuir el nivel de desequilibrio en la representación de hombres y mujeres», en http://eurlex. europa.eu/LexUriServ/LexUriServ.do?uri=OJ:C:2003:134:0003:0004:ES:PDF. Además, véase: Fernández Sánchez (2016, p. 465): «[...] el ministerio de educación [...] y el instituto de la mujer [...] señalan que las mujeres representan el $17 \%$ y el $26.59 \%$ de los estudiantes de informática e ingeniería de telecomunicaciones respectivamente. Esta infrarrepresentación de mujeres en las áreas científicas ya está presente durante la educación secundaria y postobligatoria, ya que los chicos muestran un mayor interés y elección de las materias de física y química y tecnología, mientras que las chicas por biología y sanidad [...], afirman que conocer los factores que determinan las elecciones de estudios es muy útil para el sistema educativo, los individuos y la sociedad, ya que esto ayuda a orientar mejor los objetivos de auto-realización personal y social de los estudiantes». Sobre este último aspecto véase, por ejemplo: Sáinz (2011); Manassero y Vázquez (2010).

${ }^{20}$ Esta cuestión es planteada también por Castells, (2004, pp. 159-270). El autor intenta contestar a la pregunta ¿El fin del patriarcado? (es también el título de uno de los párrafos del capítulo 4) a través un análisis de como las mujeres han sido incorporadas en el mundo laborales y al trabajo remunerado. Castells nota que «la entrada masiva de las mujeres en la mano de obra remunerada se debe, por una parte, a la informacionalización, la interconexión y la globalización de la economía y, por la otra, a la segmentación por géneros del mercado laboral, que aprovecha las condiciones sociales específicas de las mujeres para incrementar la productividad, el control de gestión y, en definitiva, los beneficios» ( $p$. 182).
} 
en cuenta los resultados presentados en el informe de la agenda 2030 para el desarrollo sostenible acerca de la igualdad de género.

Más allá de esta consideración, las TIC tienen un papel importante en el proceso de educación sobre cuándo afectan y/o benefician a la sociedad con la inserción de nuevos escenarios, formas de relación y aprendizajes (Vivanco, 2015, p. $305)^{21}$. Así que ¿cómo el género influye en el uso de las nuevas tecnologías y en la percepción que uno tiene sobre las mismas?

Es interesante indicar que esta última consideración abre las puertas a reflexionar acerca de los objetivos sobre la igualdad de género en la agenda 2030 para el desarrollo sostenible. Aquí mi intención no es, puntualmente, tratar de la enorme discusión sobre este documento ni de tratar los estudios y los datos producidos en el informe en su totalidad ${ }^{22}$. Mis propósitos principales, en esta parte del trabajo, son establecer de manera sencilla y sin pretensión alguna una evidencia de conexión con el tema de la igualdad de género, el desarrollo sostenible y el empoderamiento a partir de conocimiento lo que incluye a las TIC. Por ello, para impulsar la plena realización de los Objetivos de Desarrollo Sostenible 2030 desde el pensamiento ${ }^{23}$, parece necesario dotar a la educación de un nuevo sentido y significado pedagógico. Sin embargo, el nivel de cualificación de las personas requerido con referencia a las TIC es superior pero son las propias TIC que hoy en día asumirían un papel determinante en la creación del conocimiento social. Sin duda, para realizar las demandas de la sociedad de la información, el sistema educativo no puede quedarse al margen de esta nueva sociedad y debe adaptarse a los nuevos cambios sociales, económicos y tecnológicos. Sin duda, la tecnología juega un papel determinante en la sociedad actual, y por ello, al comentar este problema, cabe señalar que «para tener éxito en el mundo actual, marcado y dominado por la tecnología, parece necesario que cada individuo adquiera conocimientos y desarrolle competencias tecnológicas, por lo tanto las mujeres no deberían quedarse atrás» (Papastergiou, 2008, p. 595).

Esta afirmación se coloca perfectamente en línea con lo previsto, por ejemplo, en los Objetivos de Desarrollo Sostenible 2030 y en particular con el Objetivo 5: Lograr la igualdad entre los géneros y empoderar a todas las mujeres y las niñas donde se afirma que la igualdad entre los géneros no es solo un derecho humano fundamental, sino la base necesaria para conseguir un mundo pacífico, próspero y sostenible ${ }^{24}$.

Así, definir la igualdad como derecho fundamental, nos conduce a lo ya argumentado, es decir que la igualdad se caracteriza por ser un principio normativo, compuesto, por un lado, de un contenido formal y por otro lado de un contenido substancial, perfectamente compatible con la diferencia. De esta manera sigue, por ejemplo, que

Si se facilita la igualdad a las mujeres y niñas en el acceso a la educación, a la atención médica, a un trabajo decente, y una representación en los procesos de adopción de

\footnotetext{
${ }^{21}$ La autora reflexiona críticamente sobre la incorporación de las tecnologías de la información y la comunicación en la educación, cuestionando la posibilidad de valorar la diversidad en la actual tendencia homogeneizadora de las políticas educacionales y se propone contribuir con una perspectiva cultural para abordar el aprendizaje escolar con tecnologías digitales, dentro del marcos de igualdad democrática, pero con orientación a la diversidad cultural.

22 Véase: http://www.unwomen.org/es/what-we-do/post-2015

${ }^{23} \mathrm{http}: / / \mathrm{www}$.un.org/sustainabledevelopment/es/gender-equality/

${ }^{24} \mathrm{http}$ ://www.un.org/sustainabledevelopment/es/gender-equality/
} 
decisiones políticas y económicas, se estarán impulsando las economías sostenibles y las sociedades y la humanidad en su conjunto se beneficiarán al mismo tiempo ${ }^{25}$.

Esto no es una mera utopía, incrementar la presencia femenina en los varios sectores, así como en el sector TIC, es posible solamente que requiere un importante esfuerzo transformador desde el pensamiento de considerar la igualdad no solo como un principio descriptivo y/o un juicio de hechos sino también como un principio que implica necesariamente un juicio de valor. En fin, también en la relación entre las tecnologías de la información y la comunicación (TIC) y la condición de la mujer emerge que la dicotomía entre igualdad y diferencia aumenta la discriminación de género y en vez de solucionar el problema lo complica.

\section{Conclusiones}

Mediante el análisis de la primera ola del feminismo he procurado mostrar en qué sentido se caracteriza con referencia a la igualdad. En particular, se centra en lograr importantes resultados en relación con el derecho al voto, el acceso al empleo, la educación y la libertad de elección en caso de aborto, es decir, en todos los campos en los que es posible lograr la igualdad formal o eliminar la falta de igualdad formal. Más allá de las discusiones acerca de las funciones que cumple la igualdad formal, he subrayado también que las reformas en el derecho laboral y familiar se han basado en la identidad del tratamiento sin tener en cuenta las condiciones reales de las mujeres y las relaciones de poder dentro de la familia, por lo que ignoran las diferencias y tratar a todas las personas de manera igual puede dar lugar a discriminaciones inesperadas (Gianformaggio, 1993; Ferrajoli, 1993; Gerhard, 1997). Así,

el feminismo de la igualdad se ha traducido en la exigencia de tratamiento igual, en el sentido, por un lado, de la eliminación de las discriminaciones manifiestas entre mujeres y hombres, por otro lado, de la constitución de las mujeres como sujetos dotadas de plena autonomía que rechazan las normas proteccionistas; el feminismo de la diferencia se manifestó en las solicitudes de tratamiento especial, que lograra una igualdad sustancial a través de la valorización de las diferencias, destacando la falsa neutralidad del derecho (Facchi, 1999, p. 135).

Sin embargo, también en contra del feminismo de la diferencia es posible formular algunas críticas. En primer lugar, la afirmación de los tratamientos especiales basados en el género podría abrir nuevamente las puertas a las políticas de protección de la mujer que han caracterizado aquella propia sociedad conservadora, que reconoce una cierta propensión (naturaleza) de las mujeres por una actividad en lugar de otra, combatida por el feminismo en sus primeras manifestaciones. Pero esto no significa que el feminismo de la diferencia no tenga el mérito de subrayar de manera más evidente que el derecho es un instrumento masculino y por ello, no ser capaz de ofrecer una protección adecuada a las mujeres. Por tanto, ha construido un constante debate sobre la utilidad y la oportunidad de usar el derecho, comparando tanto en un nivel teórico como en un nivel concreto, por un lado, la desconfianza en el derecho como una técnica sexuada, y por otro lado la búsqueda de un derecho femenino (Facchi, 1999, p. 139). Finalmente, hay también que subrayar que dentro del feminismo que se origina en la exaltación de las diferencias entre la mujer y el hombre, se destaca un pensamiento feminista más radical que apoya las diferencias dentro de la diferencia, con la consecuencia que las teorías elaboradas por una parte del feminismo - por ejemplo la parte blanca del feminismo- no son aceptables para los otros grupos étnicos (como por ejemplo por las mujeres de color) cuyos problemas se refieren a condiciones sociales y económicas además que culturales radicalmente

\footnotetext{
25 Ibidem.
} 
diferentes (Abbagnano, 2012, p. 471). En conclusión, aunque sencillamente, mediante este análisis emerge que la dicotomía igualdad-diferencia ha empantanado para un largo tiempo el debate dentro del feminismo que será superado tal vez definitivamente solo con la tercera ola, es decir con el feminismo radical, que tiende a subvertir aquellas relaciones sexuales y sociales en las que hay una opresión de los hombres en contra de las mujeres. Por ejemplo, Catherine MacKinnon, una de las principales exponentes de esta ola del feminismo, no plantea la cuestión de si el derecho debería tratar a las mujeres y a los hombres de manera igual o diferente, sino que el propio derecho no se convierta en un instrumento de opresión y subordinación. Así en ese sentido parece claro que la contraposición teórica entre el feminismo de la igualdad y el feminismo de la diferencia, que surgió principalmente en América, podría superarse proponiendo una forma de lectura que intenta conciliar las dos alternativas; puesto que el extremismo de las dos tesis solo funcionaría en el nivel teórico, ciertamente no en el nivel práctico-real.

\section{Bibliografía}

Abbagnano N. (2012). Dizionario di filosofia. Torino, Italia: Utet.

Area M. (2009). Introducción a la tecnología educativa. Universidad de la Laguna, España: Creative Commons.

Area M., Gros B. y Marzal M. A. (2008). Alfabetizaciones y tecnologías de la información y la comunicación. Madrid, España: Editorial Síntesis S. A.

Benhabib S. (2006). Las reinvindicaciones de la cultura: igualdad y diversidad en la era de la globalización. Buenos Aires, Argentina: Katz.

Boccia M. L. (2002). La differenza politica. Donne e cittadinanza. Milano, Italia: II Saggiatore.

Bock G., James S. (1992). Beyond Equality and Difference. London-New York, UKUSA: Routledge.

Bono P., Kemp S. (1991). Italian Feminist Thought. Oxford, UK: Blackwell.

Botti C., Etica e differenza di genere. Recuperado de

http://www.treccani.it/scuola/tesine/maschile e femminile/2.html.

Casadei T., (ed). (2015). Donne, diritto, diritti. Prospettive del giusfemminismo. Torino, Italia: Giappichelli.

Castaño C. (2008). La segunda brecha digital. Madrid, España: Cátedra.

Castells M. (2004). La era de la información. Economía Sociedad y Cultura, El poder de la identidad, vol. II. Ciudad de México, México: Siglo XXI Editores.

Cavarero A. (1987). Per una teoria della differenza sessuale, in Diotima. II pensiero della differenza sessuale. Milano, Italia: La Tartaruga.

Cavarero A. y Restaino F. (1999). Le filosofie femministe. Torino, Italia: Paravia.

Cavarero A. y Restaino F. (2002). Le filosofie femministe. Milano, Italia: Mondadori.

Cockburn C. (1983). Brothers: Male dominance and technological change. Londres, UK: Pluto Press.

Cuono M. y Gargiulo E. (2017). Emergenza, crisi, sicurezza. Decisioni extra-ordinarie tra governo centrale e amministrazioni locali, Diritto\&Questioni Pubbliche, volumen 17 (núm. 2), 15-36.

Evans J. R. (1977). The feminists: women's emancipation movements in Europe, America, and Australia, 1840-1920. Londres, UK: Routledge.

Facchi A. (2004). Diritti delle donne tra universalismo e particolarismo. Introduzione, Ragion Pratica, volumen 23, (núm. 2), 325-338.

Facchi A. (2007). Breve storia dei diritti umani. Bologna, Italia: II Mulino.

Facchi A., (1999). II pensiero femminista, sul diritto: un percorso da Carol Gilligan a Tove Stang Dahl. En G. Zanetti, Filosofi del diritto contemporaneo, (pp. 129-153). Milano, Italia: Raffaello Cortina Editore.

Fernández Sánchez A. (2016). La dirección escolar ante el reto de implantar un plan 
TIC con perspectiva de género. En M. Blanco, R. San Segundo, Investigación joven con perspectiva de género, Libro de Actas del I Congreso de jóvenes investigadorxs con perspectiva de género celebrado en la Universidad Carlos III de Madrid, Getafe, 16 y 17 de junio de 2016. Getafe, España: Instituto de Estudios de Género, Universidad Carlos III de Madrid.

Ferrajoli L. (1993). La differenza sessuale e le garanzie dell'uguaglianza, Democrazia e Diritto, (núm. 2), 50-53.

Ferrajoli L. (1995). Derecho y Razón. Teoría del garantismo penal. Madrid, España: Trotta.

Ferrajoli L. (2015). II principio di uguaglianza e la differenza di genere, Giudicedonna.it, (núm.3), pp. 1-14.

Fineman M. A. (2005). Feminist Legal Theory, Journal of Gender, Social Policy and the Law, volumen 13, (núm. 1), 13 - 23.

Friedan B. (2009). La mística de la feminidad. Madrid, España: Ediciones Cátedra.

Gatens M. (1991). Feminism and Philosophy. Perspectives on Difference and Equality. Cambridge, UK: Polity Press.

Gerhard U. (1997). Femminismo e diritto: verso una concezione femminista e contestualizzata dell'uguaglianza, Ragion Pratica, (núm. 8), 53-62.

Gianformaggio L. (1993). Eguaglianza e differenza: sono veramente incompatibili?. En G. Bonacchi, A. Groppi (ed.), Il dilemma della cittadinanza. Diritti e doveri delle donne, (pp. 214-240). Roma-Bari, Italia: Laterza.

Gianformaggio L. (1993). Identity, Equality, Similarity and the Law, Rechtsteorie, volumen $15,121-134$.

Gianformaggio L. (1995). Filosofia e Critica del Diritto. Torino, Italia: Giappichelli.

Gianformaggio L. (1996). Eguaglianza formale e sostanziale: il grande equivoco, II Foro Italiano, (núm. 1), 1961-1976.

Gilligan C. (1982). In a Different Voice. Cambridge, Massachusetts, USA: Harvard University Press.

Grant Bowman C., Schneider E. M. (1998). Feminist Legal Theory, Feminist Lawmaking and the Legal Profession, Fordham Law Review, volumen 67, (núm. 2), 249-271.

Haraway D. (1991). Manifiesto Ciborg. El sueño irónico de un lenguaje común para las mujeres en el circuito integrado. Santa Cruz, California, USA: University of California.

Harding S. (1998). ¿Existe un método feminista? En Barta, Eli, (coord.), Debates en torno a una metodología feminista. Xochimilco, México: Universidad Autónoma Metropolitana.

Irigaray L. (1976). Speculum. L'altra donna (1974). Milano, Italia: Feltrinelli.

Jaggar A. (1983). Feminist Politics and Human Nature, Rowman and Allanheld. New Jersey, USA: Rowman\&Allanheld.

Kymlicka W. (1995). Filosofía política contemporánea: una introducción, trad. R. Gargarella. Barcelona, España: Ariel.

Libreria delle donne di Milano (ed.) (1987). Non credere di avere diritti. Torino, Italia: Rosenberg e Sellier.

Lonzi C. (1974). Sputiamo su Hegel. La donna clitoridea e la donna vaginale, Rivolta femminile. Milano, Italia: Editorial.

Mackinnon C. (1983). Feminism, Marxism, Method and the State: toward feminist jurisprudence, Signs, volumen 8, (núm. 4), 635-658.

Mackinnon C. (2014). Feminismo inmodificado. Discursos sobre la vida y el derecho. Buenos Aires, Argentina: Siglo XXI Editores.

Manassero M. A, Vázquez A. (2010). Perfiles actitudinales de la elección de ciencias en secundaria según el sexo y el tipo de educación, Revista Electrónica de Enseñanza de las Ciencias, volumen 9, (núm. 1), 242-260.

Mancina C. (2002). Oltre il femminismo. Le donne nella società pluralista. Bologna, Italia: II Mulino. 
Martínez Álvaro L. (2016). Patriarcado 2.0: Retención del talento femenino en las empresas tecnológicas. En M. Blanco, R. San Segundo, Investigación joven con perspectiva de género, Libro de Actas del I Congreso de jóvenes investigadorxs con perspectiva de género celebrado en la Universidad Carlos III de Madrid, Getafe, 16 y 17 de junio de 2016. Getafe, España: Instituto de Estudios de Género, Universidad Carlos III de Madrid.

Morondo Taramundi D. (2004). II dilemma della differenza nella teoria femminista del diritto. Pesaro, Italia: Aras Edizioni.

Muraro L. (1991). L'ordine simbolico della madre. Roma, Italia: Editori Riuniti.

Nicholson L. (1997). Second Wave: A Reader in Feminist Theory. New York, USA: Routledge.

Nussbaum M. (2002). Rawls and feminism. En S. Freeman, The Cambridge companion to Rawls (pp. 488-520). Cambridge, UK: Cambridge University Press.

Okin S. M. (1989). Justice, Gender, and the Family. New York, USA: Basic Books.

Papastergiou M. (2008). Are computer science and information technology still masculine fields? High school students' perceptions and career choices, Computers \& Education, volumen 51, (núm. 2), 594-608.

Pérez Luño E. (2007). Las dimensiones de la igualdad. Madrid, España: Dykinson.

Restaino F. (1999). II pensiero femminista. Una storia possibile. En F. Restaino y A. Cavarero. Le filosofie femministe (pp. 3-54). Torino, Italia: Paravia.

Sáinz M. (2011). Factors which influence girls' orientations to ICT subjects in schools. Evidence from Spain, International Journal of Gender, Science and Technology, volumen 3 (núm. 2), 387-406.

Scott J. W. (1988). Deconstructing Equality Versus Difference: or the Uses of Poststructuralist Theory of Feminism, Feminist Studies, volumen 14, (núm. 1), 3250.

Scott J. W. (1995). Deconstructing Equality Versus Difference: or the Uses of Poststructuralist Theory for Feminism. En: Frances Olsen (ed.), Feminist legal theory, (pp. 261-280). New York, USA: New York University Press.

Sosa T. (21 junio, 2013). Las mujeres y las TIC: Tecnologías de la Información y la Comunicación a su servicio. Recuperado de https://palabrademujer.wordpress.com/?s=TIC

Stamile N. (2015). Uguaglianza e ragionevolezza. En L. R. Corrado (ed.), Le uguaglianze: diritti, risorse, sfide per il futuro (pp. 11-24). Milano, Italia: Hòrisma, Editore, Ledizioni.

Stamile N. (2017). Una misteriosa amistad: la relación de las mujeres con la Tecnologías de la Información y Comunicación, ponencia presentada al XI Conferencia Internacional de derecho e informática de La Habana 2017 - VII Congreso iberoamericano de investigadores y docentes de derecho e informática (CIIDDI) celebrado los días 15-19 de mayo de 2017 en La Habana (Cuba).

Stein L. W. (1993). Living with the Risk of Backfire: A Response of the Feminist Critiques of Privacy and Equality, Minnesota Law Review, volumen 77, (núm. 1), pp. 1153-1191.

Vilardo V. (8 Octubre, 2008). Mujeres, TIC y transparencia - Tecnología - publicado en Mujeres en Red. Recuperado de http://www.mujeresenred.net/spip.php?article1762.

Vivanco G., (2015). Educación y tecnologías de la información y la comunicación. ¿es posible valorar la diversidad en el marco de la tendencia homogeneizadora?, Revista Brasileira de Educação, volumen 20, (núm. 61), pp. 297-315.

Volpe G. (1977). L'ingiustizia delle leggi. Studi sui modelli di giustizia costituzionale. Milano, Italia: Giuffrè.

Young M. (2000). La justicia y la política de la diferencia. Madrid, España: Ediciones Cátedra. 